\title{
The QCD scattering amplitude from area behaved Wilson loops
}

\author{
Y. Makeenko \\ Institute of Theoretical and Experimental Physics, \\ B. Cheremushkinskaya 25, 117218 Moscow, Russia* \\ P. Olesen \\ The Niels Bohr International Academy, \\ The Niels Bohr Institute, \\ Blegdamsvej 17, 2100 Copenhagen Ø, Denmark ${ }^{\dagger}$
}

(Dated: January 24, 2012)

\begin{abstract}
We explicitly construct the dominant saddle-point trajectory in the sum-over-path representation of meson scattering amplitudes in large $N$ QCD for area-behaved Wilson loops and show that it dominates in the Regge regime. The graphic representation of the leading trajectory is very similar to the diagrams widely used to illustrate meson scattering.

PACS numbers: 11.25.Tq, 12.38.Aw, 11.15.Pg

Keywords: large N QCD, Wilson loop, meson scattering amplitude, Regge regime, reparametrization path integral, saddle point trajectory
\end{abstract}

In this Letter we study the large $N$ QCD scattering amplitudes of colorless composite quark operators given as a path integral over Wilson loops $[1,2]$. We find the most important paths by means of a double saddle point method. A geometric representation of these paths is very similar to the dual diagrams which for many years have been used to illustrate meson scattering amplitudes.

Previously we have considered [3] the large $N$ (or quenched) QCD meson scattering amplitude, using the connection between this amplitude and the Wilson loop in the case where the loop is well represented by the area behavior. This QCD scattering amplitude then turns out to be the Regge limit of the usual Veneziano amplitude in the cases when the number of external particles is large, or when the quark mass is small relative to the QCD- $\Lambda$. As discussed in [3] this is a prediction for near forward scattering at high energies, that is associated with large distances in the relevant functional integral. The goal of this Letter is to demonstrate this by explicitly constructing the saddle point trajectory.

The relation between the off shell $M$ meson scattering amplitude $G$ and the Wilson loop occurs as a functional integral [3],

$$
\begin{aligned}
G\left(p_{1}, \ldots, p_{M}\right) \propto & \prod_{i=1}^{M-1} \int_{0}^{\phi_{i+1}} d \phi_{i} \int \mathcal{D} x(\phi) e^{i \int d \phi p(\phi) \dot{x}(\phi)} \\
& \times W(x(\phi)), \quad x(0)=x(2 \pi),
\end{aligned}
$$

in the limit of large $M$ or small quark masses, when the spin dependence factorizes. In this expression $W(x(\phi))$ is the Wilson loop defined on the boundary curve $x(\phi)$, and the function $p(\phi)$ is piecewise constant, defined by

$$
p(\phi)=q_{i} \text { for } \phi_{i}<\phi<\phi_{i+1},
$$

\footnotetext{
*Electronic address: makeenko@itep.ru

†Electronic address: polesen@nbi.dk
}

where the momenta $q_{i}$ are related to the external momenta $p_{i}$ 's by

$$
p_{i}=q_{i-1}-q_{i},
$$

thus ensuring conservation of the energy-momentum. In particular we note that Eq. (2) implies the very useful result

$$
\dot{p}(\phi)=-\sum_{i} p_{i} \delta\left(\phi-\phi_{i}\right)
$$

Also, in Eq. (1) the Wilson loop is required to be bounded by the closed curve $x^{\mu}(\phi)$.

In the basic relation (1) we now want to insert the area behavior $W \sim \exp (-$ area), where the area is measured in terms of the string tension. If we insert the area as the usual square root of the determinant of the extrinsic metric and minimize this, the functional path integral in (1) becomes impossible to perform because it depends on surface variables instead of the paths in (1) and also because of the highly non-linear character of the integration. We therefore suggested [3] to use Douglas' expression for the minimal area [4],

$$
\begin{aligned}
A[x]=\frac{-1}{4 \pi} \int_{0}^{2 \pi} d \theta_{1} \int_{0}^{2 \pi} d \theta_{2} \dot{x}\left(\theta_{1}\right) \dot{x}\left(\theta_{2}\right) \\
\times \log \left[1-\cos \left(\phi\left(\theta_{1}\right)-\phi\left(\theta_{2}\right)\right)\right] .
\end{aligned}
$$

This integral should be minimized with respect to the reparametrization function $\theta(\phi)$, subject to the requirement $\theta\left(\phi_{i}\right)=\phi_{i}$. We should therefore look for a saddle point with respect to the reparametrization.

The expression (5) is known from the derivation of the string disk amplitude in the Polyakov formulation, where it appeared as the boundary action after integration over fluctuations of an open string with the Dirichlet boundary conditions at the ends. It is less known that the crucial role is then played by the path integral over the 
boundary metric (the same as the path integral over the reparametrizations), which is dominated for large loops by the saddle point in hands, reproducing the minimal area. However, for small loops $W(x(\phi))$ in Eq. (1) is described by QCD perturbation theory, rather than by the string disk amplitude, thus leading us to the understanding [5] of QCD string as an effective rather than fundamental string. It makes sense only for long strings and we shall soon justify by an explicit calculation that the double saddle point we consider is indeed associated with large loops or long QCD string.

Douglas' integral (5) is apparently well suited for insertion in the path integral (1), where it leads to an apparent Gaussian integration. However, this point of view is an illusion - the non-linearity is still with us, because we have to minimize the integral (5) with respect to reparametrizations $\theta(\phi)$ or the inverse $\phi(\theta)$. As shown by Douglas, this leads to the requirement

$$
\dot{x}\left(\theta_{1}\right) \int_{0}^{2 \pi} d \theta_{2} \dot{x}\left(\theta_{2}\right) \cot \left(\frac{\phi\left(\theta_{1}\right)-\phi\left(\theta_{2}\right)}{2}\right)=0 .
$$

This condition is highly non-linear!

We now proceed to tackle this problem by a two step mechanism: first we find the saddle point in the $x$-integral for any reparametrization (justified because we seek a large minimal area), and then we require that for this saddle point the reparametrization must satisfy the condition (6). Thus we search for a double saddle point.

From Eq. (1) the saddle point condition for large area or, alternatively, large string tension is

$i p^{\mu}(\theta)+\frac{\sigma}{2 \pi} \int_{0}^{2 \pi} d \theta^{\prime} \dot{x}^{\mu}\left(\theta^{\prime}\right) \log \left[1-\cos \left(\phi(\theta)-\phi\left(\theta^{\prime}\right)\right)\right]=0$

for $W \sim \exp (-\sigma A)$, where $\sigma$ is the string tension. This equation can easily be solved by noting that the log is a Green function with a simple inverse,

$$
\begin{aligned}
G(\phi) & \equiv \frac{-1}{2 \pi} \log (2-2 \cos \phi), \\
G^{-1}(\phi) & =-\ddot{G}(\phi)=\frac{-1}{2 \pi(1-\cos \phi)} .
\end{aligned}
$$

Inverting (7) we therefore get

$$
x^{\mu}(\theta)=\frac{i}{2 \pi \sigma} \int_{0}^{2 \pi} d \theta^{\prime} \dot{p}^{\mu}\left(\theta^{\prime}\right) \log \left[1-\cos \left(\phi(\theta)-\phi\left(\theta^{\prime}\right)\right)\right] .
$$

Note that this saddle point $x$ is imaginary, as is to be expected from the occurrence of $i p x$ in (1). Next we can use Eq. (4) to obtain

$$
x^{\mu}(\theta(\phi))=\frac{-i}{2 \pi \sigma} \sum_{j} p_{j}^{\mu} \log \left[1-\cos \left(\phi-\phi_{j}\right)\right] .
$$

We note the subtle point that in principle the right-hand side depends on the reparametrization, but the left-hand side is independent! Thus, the saddle point does not care about $\theta(\phi)$, i.e. the reparametrization can be any function.

We now have to show that the saddle point (10) actually satisfies the non-linear Douglas condition (6). From (10) we have

$$
\dot{x}^{\mu}(\theta)=\frac{-i}{2 \pi \sigma} \dot{\phi}(\theta) \sum_{j} p_{j}^{\mu} \cot \left(\frac{\phi(\theta)-\phi\left(\theta_{j}\right)}{2}\right) .
$$

Inserting this on the left-hand side of Eq. (6), we obtain

$$
\dot{x}(\theta) \sum_{j} p_{j} \int_{0}^{2 \pi} d \phi^{\prime} \cot \left(\frac{\phi^{\prime}-\phi_{j}}{2}\right) \cot \left(\frac{\phi^{\prime}-\phi(\theta)}{2}\right) \stackrel{?}{=} 0 .
$$

The left hand-side can be easily evaluated if we perform a partial integration and use Eqs. (8a) and (8b),

$$
\begin{aligned}
& \text { left-hand side of }(12) \\
& \propto \dot{x}(\theta) \sum_{j} p_{j} \int_{0}^{2 \pi} d \phi^{\prime} G\left(\phi^{\prime}-\phi_{j}\right) G^{-1}\left(\phi^{\prime}-\phi(\theta)\right) \\
& =\dot{x}(\theta) \sum_{j} p_{j} \delta\left(\phi(\theta)-\phi_{j}\right)
\end{aligned}
$$

In order to satisfy the Douglas condition (6) we therefore need to have

$$
\sum_{i} p_{i} p_{j} \cot \left(\frac{\phi_{i}-\phi_{j}}{2}\right)=0
$$

for each value of $j$. Among these equations only $M-3$ are independent because of the projective symmetry, thus fixing $M-3$ integration variables $\phi_{i}$ 's as a consequence of the fact that we are looking for a double saddle point. The remaining three variables can be fixed arbitrarily.

Instead of the angle $\phi$ ranging between 0 and $2 \pi$ it is often convenient to use

$$
r=-\cot (\phi / 2)
$$

which ranges from $-\infty$ to $+\infty$. The saddle point solution (10) then becomes

$$
x^{\mu}(u(r))=\frac{-i}{\pi \sigma} \sum_{j} p_{j}^{\mu} \log \left|r-r_{j}\right| .
$$

Here $u(r)$ is any reparametrization of the boundary curve.

Since the trajectory (16) is the saddle point of the amplitude (1), it can be approximated by the value of the integrand at this saddle point. We thus obtain [3]

$$
\begin{gathered}
e^{-\sigma A[x]+i \int p(r) \dot{x}(r) d r} \mid \text { saddle point value } \\
=e^{(1 / 2 \pi \sigma) \sum_{i, j} p_{i} p_{j} \log \left|r_{i}-r_{j}\right|}
\end{gathered}
$$

which coincides with the saddle point approximation to the integral over the Koba-Nielsen variables in the $M$ point Veneziano amplitude. 
This stems from the fact that the Douglas procedure leads to a determination of the parameters $r_{i}$ 's in a similar way how we have seen this in Eq. (14) for the corresponding parameters $\phi_{i}$ 's. Proceeding as before we obtain $[6]$

$$
\sum_{i} \frac{p_{i} p_{j}}{r_{i}-r_{j}}=0
$$

The meaning of this condition is easy to understand: on the right-hand side of Eq. (17) the condition (18) corresponds to the extremum, generally including the Regge limit, and this extremum is thus exactly the minimal surface entering the Wilson loop in QCD!

It is instructive to see how the curve (16) looks like for the four point function in the center of mass frame. Because we deal with the case where both Mandelstam's variables $s$ and $t$ are negative, this is associated with a scattering process in $u$-channel. In view of the projective symmetry we can set three out of four $r_{j}$ 's equal to certain typical values, keeping in mind that one such contour belongs, in fact, to a whole family of contours, which are equally important. The projective invariant ratio $\left(r_{2}-r_{1}\right)\left(r_{4}-r_{3}\right) /\left(r_{3}-r_{1}\right)\left(r_{4}-r_{2}\right)$ is then fixed by Eq. (18) to be $t /(t+s)$. We plot the corresponding contour in Fig. 1 for $s / t=10$ in the units of $i \sqrt{u} / 2 \pi \sigma$. The contours are quite normal for not too small $t / s$ and look pretty much similar to dual diagrams used to illustrate the scattering of mesons. For $r \rightarrow r_{j}$ we have

$$
x^{\mu}(u(r)) \rightarrow \frac{-i}{\pi \sigma} p_{j}^{\mu} \times \text { regularizing factor. }
$$

Actually, Fig. 1 is obtained without any regularization, which is implemented by Mathematica itself. The transverse size of the contour shrinks when $t / s \rightarrow 0$ in spite of the fact that the minimal area

$$
\sigma S_{\min }(C)=\alpha^{\prime} t \log \frac{s}{t}, \quad \alpha^{\prime}=\frac{1}{2 \pi \sigma},
$$

can be large even for small $t$. For the self-consistency of our approach this transverse size should be larger than the confinement scale $1 / \Lambda_{\mathrm{QCD}} \sim 1 / \sqrt{\sigma}$.

A very interesting question is as to how we can make the saddle point trajectory (16) to be real. This happens in the physical domain of $s$-channel, when $s>0, t<0$ and $u<0$, so that $-i \sqrt{u}=\sqrt{s}$. We may think about this as an analytic continuation in $s$ from negative to positive values, keeping $|s| \gg-t$ all the way. The area of such a surface will be a complex number because of Minkowskispace kinematics but with a negative real part, as is seen from Eq. (20), what is essential in our consideration for it to dominate in the path integral.

There is a number of well-known reasons why the amplitude (17) cannot be right in all kinematical regions of QCD. For example, for large transverse momenta the decay should be power like, in contrast to the dual models, and furthermore, QCD has of course no tachyon. In this connection it should be remembered that the area

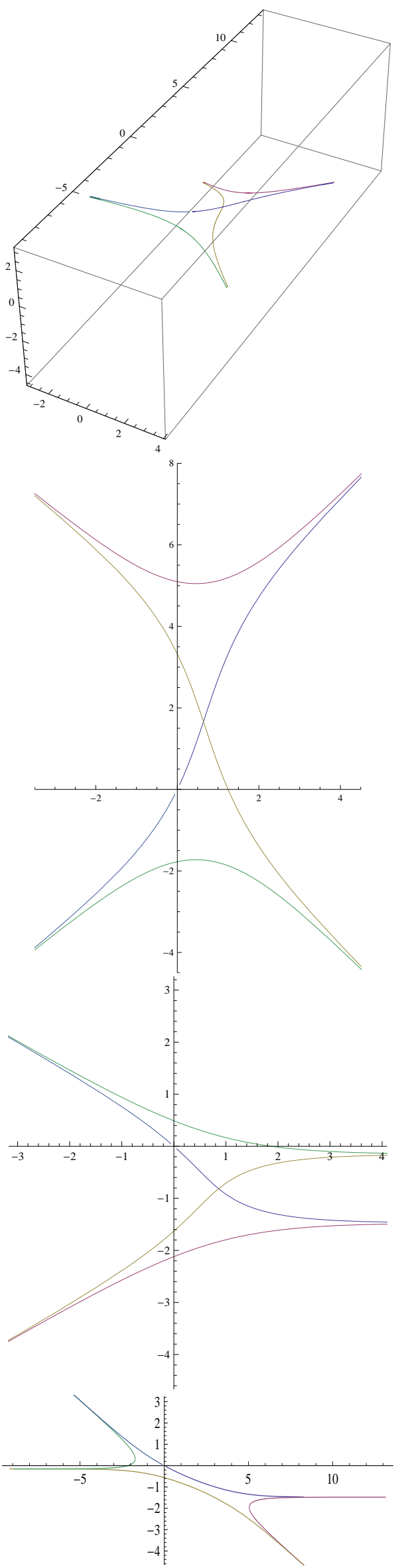

FIG. 1: Saddle-point trajectory (16) for $t / s=0.1$ : $3 \mathrm{D}$ view and its projections in 0,$1 ; 0,2$ and 1,2 planes, respectively. 
behaved Wilson loop is quite small, so it may only dominate in restricted kinematical regions. For large transverse momenta the amplitude corresponding to Eq. (17) decays exponentially, so the power behaved perturbative behavior of $W$ is much more important for large transverse momenta. We therefore expect the area behavior to dominate only at large energies in the near forward direction. Since the tachyon is a low energy phenomenon [5] it is therefore not relevant in our work.

From Eq. (16) we can obtain the equation for the minimal surface because $x$ has to be harmonic,

$x^{\mu}(r, y)=\frac{-i}{\pi \sigma} \sum_{j} p_{j}^{\mu} \log \left|z-r_{j}\right|, \quad z=r+i y, \quad y \geq 0$.

This expression is the imaginary part of the analytic function

$$
\frac{-i}{\pi \sigma} \sum_{j} p_{j}^{\mu} \log \left(z-r_{j}\right)
$$

whose real part

$$
\frac{1}{\pi \sigma} \sum_{j} p_{j}^{\mu} \arctan \frac{r-r_{j}}{y}
$$

is also harmonic and describes [6] the minimal surface bounded by a step function

$$
\frac{1}{\sigma} \sum_{j} p_{j}^{\mu} \Theta\left(r-r_{j}\right) \Theta\left(r_{j+1}-r\right)
$$

with $\Theta$ being the Heaviside step function. It is of course related to the imaginary part by the Cauchy-Riemann equations, which in turn are related to a T-duality map $[7]$.

The induced metrics of the surfaces (21) and (23) differ only by the signs. Moreover, if we were go beyond the saddle point approximation, their transverse quantum fluctuations can be similarly taken into account by a conformal mapping of the upper half-plane onto a rectangle, associated with the worldsheet parametrization. We may expect therefore that we shall obtain for (21), like in [6] for (23), the linear Regge trajectory

$$
\alpha(t)=\frac{d-2}{24}+\alpha^{\prime} t
$$

in the semiclassical approximation for arbitrary dimension $d \geq 2$.

The expression (17) leads to straight line Regge trajectories, when the values of $r_{i}$ 's obey Eq. (18). The prediction is thus that this should occur at high energies and moderate transverse momenta, in accordance with experimental results for $\mathrm{SU}(3)$. Also our results are valid in other dimensions than four, for example in two dimensions, where 't Hooft [8] found that the mass spectrum for large masses is a linear function of an integer quantum number. For smaller masses there is a deviation from the straight line. In two dimensions the Wilson loop is area behaved everywhere, but our calculation only includes the saddle point. For smaller areas there would be corrections to what we found.

The fact that the minimal surface (21) is associated with the Regge limit of the Koba-Nielsen amplitudes is known since early days of string theory [9], though the reparametrization of the boundary was not taken into account that time. To get it within our approach, it was crucial that the path integral (1) is dominated by the saddle point trajectory (16) whose shape is not sensitive to the choice of the reparametrizing function $u(r)$. It is also worth mentioning another minimal surface of the form of a helicoid that was associated in Ref. [10] with the reggeization of quark-antiquark within the AdS/CFT correspondence in a confining background. It will be interesting to understand how these two are related. However, we emphasize once again that our approach is based solely on the representation of the Wilson loop via the boundary functional (5) and does not use explicitly a surface representation.

As a conclusion we see that the large $N$ meson scattering amplitude expressed in terms of a path integral is dominated by a double saddle point for large energies and moderate transverse momenta. The graphic representation of the leading trajectories is similar to the dual diagrams used to illustrate meson scattering.
[1] K. G. Wilson, Phys. Rev. D 10 (1974) 2445.

[2] Y. M. Makeenko and A. A. Migdal, Nucl. Phys. B 188 (1981) 269.

[3] Y. Makeenko and P. Olesen, Phys. Rev. Lett. 102 (2009) 071602, arXiv:0810.4778 [hep-th]; Y. Makeenko and P. Olesen, Phys. Rev. D 80 (2009) 026002, arXiv:0903.4114 [hep-th].

[4] J. Douglas, Trans. Amer. Math. Soc. 33 (1931) 263.

[5] P. Olesen, Phys. Lett. B 160 (1985) 144.

[6] Y. Makeenko, Phys. Rev. D 83 (2011) 026007,
arXiv:1012.0708 [hep-th].

[7] P. Caputa and S. Hirano, arXiv:1108.2381 [hep-th].

[8] G. 't Hooft, Nucl. Phys. B 75 (1974) 461.

[9] C. S. Hsue, B. Sakita and M. A. Virasoro, Phys. Rev. D 2 (1970) 2857.

[10] R. A. Janik and R. Peschanski, Nucl. Phys. B 625 (2002) 279, arXiv:hep-th/0110024; M. Giordano and R. Peschanski, JHEP 1110 (2011) 108, arXiv:1105.6013 [hep-th]. 